\title{
Day and Assessment Training Technology Centres
}

\author{
Geoffrey Busby MBE. D.UNIV (MIDDX) MA FBCS CEng \\ GEC Computer Services Ltd,. \\ Great Baddow, Chelmsford \\ ENGLAND
}

\begin{abstract}
Modern technology affords opportunities to severely physically and sensory disabled people that previously would have been out of the question. In this paper I am not going to discuss the opportunities mentioned above, time doesn't permit, but rather the environment of my concept of the ideal environment in which such opportunities can be explored. Essentially it's a description of a planned Centre which I am hoping to set up in S.E. London.
\end{abstract}

\section{Mission Statement}

To provide Outreach Centres across the UK where people with disabilities and those, in or entering disability professions, will be afforded the opportunity to reach their full potential.

A full range of activities will be offered through the medium of computer technology. Such activities will enable people with disability to undertake programmes of recreation rehabilitation, learning and computer applications, allowing people with disabilities to liberate their latent skills.

In short every Compaid Outreach Centre will aim to secure the lasting economic, social and physical regeneration of its surrounding locations with particular reference to empowering people with disabilities.

\section{Objectives}

* Be centres of excellence where people with all types of disability can, through the use of technology, reach their full potential.

* Be centres where people with disabilities can come to try out technical solutions in order to ascertain which is best for them.

* Be centres of excellence in disability awareness.

* The centres will provide respite care. 
* A vehicle for students/practitioners to gain practical experience.

* Provide relief for carers.

* Be a resource to local commerce.

\section{Services Provided}

* Day attendance with access to recreational and rehabilitational use of computers.

* Short courses on work related use of computers and related technology.

* Employment counsel and advice.

* Professional assessments measuring the potential of adaptive technology.

* Formal, qualification-led, courses related to computing, and employment.

* Office services (document production etc).

* Computing and IT consultancy.

* Basic care services.

* Training of trainers/carers.

\section{Choice of Location}

The first Centre will be in East London. There are a few organisations in England, similar to the proposed Centre, however on a first survey, none exists to service East London, or more generally the densely populated area of S.E. England. In addition the Royal London Hospital is the London and S.E. Centre for head injuries and their treatment - this being the type of activity which the centre can work with. Moreover at the current time there is an over-supply of business premises in the East London area, which should enable the proposed Centre to find attractively priced (or even free) space.

Many of the larger commercial, and governmental, organisations based in East London, have a positive policy on disability, and on employment/disability issues. These organisations are a source of both funding, volunteer resources across a range of skills and a natural outlet for clients who wish to progress into mainstream employment. 


\section{Relationship to Europe}

In the middle term, as project activities grow, and the Centre is seen to be establishing a national reputation. The accessibility of East London to City Airport becomes relevant as it opens up one of the ways by which the Centre would grow its European and International reputation.

\section{Physical shape of centre}

The two diagrams appended show firstly, the concept of how the Centre will work, and secondly, a detailed layout of the facilities.

\section{Concept Diagram}

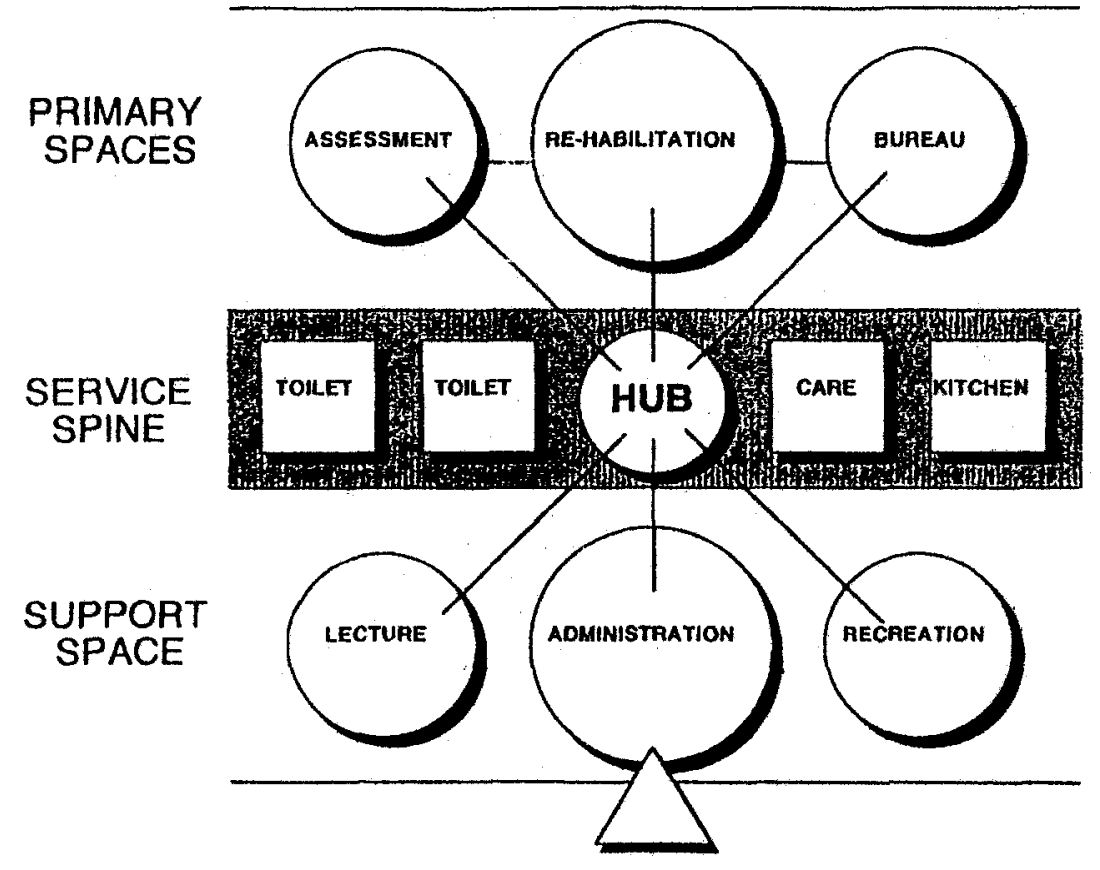




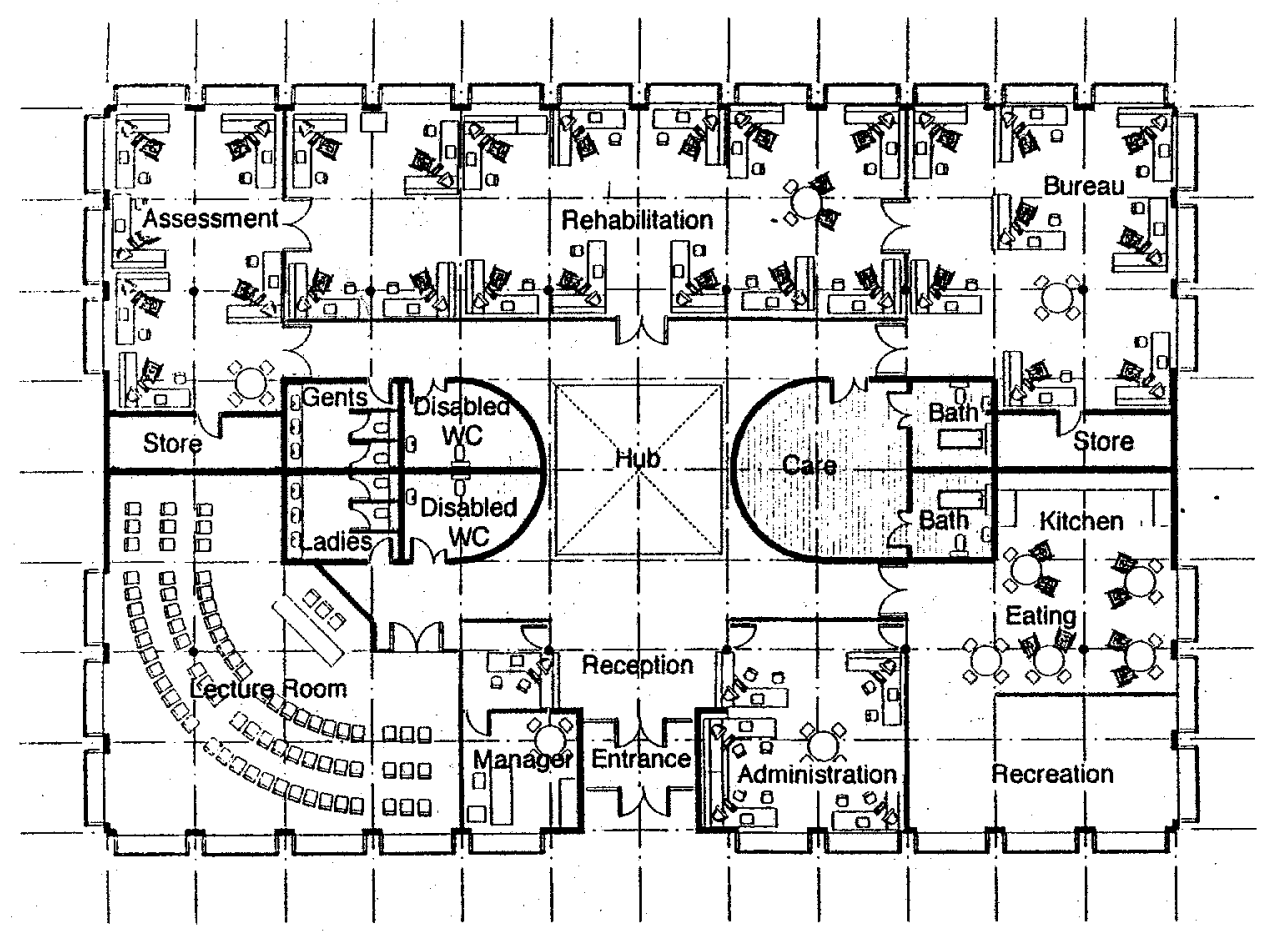

From the diagram you will see that $I$ intend to offer three main areas of service which, I believe is necessary to make up the whole.

Some of you may be surprised to see the high level of care we intend to offer, the reason for this is two fold. Firstly in Great Britain since 1992 there has been a philosophy of integrating people with all types of disability into the community. This system, "Care in the Community", has suffered from our Government failing to recognise the financial expenditure required. Secondly the outcome has been unfortunate for many people, particularly those with mental illnesses, who have found themselves residing in inappropriate accommodation without any assistance and close relations/friends of physically disabled people are finding themselves taking on an increasing care roll. I therefore hope the services offered at the Centre will overcome part of this problem while at the same time introduce such clients to technology and possibly awaken their awareness to their own potential. 